\title{
A dynamic Lagrangian frequency-time method for the vibration of dry-friction-damped systems
}

\author{
S. Nacivet ${ }^{\mathrm{a}}$, C. Pierre ${ }^{\mathrm{b}}$, F. Thouverez ${ }^{\mathrm{a}, *}$, L. Jezequel ${ }^{\mathrm{a}}$ \\ ${ }^{a}$ Laboratoire de Tribologie et Dynamique des Systèmes, Dept. de Mecaniques des Solides, Ecole Centrale de Lyon, \\ Batiment E6, 36 Avenue Guy de Collongue, BP163, 69131 Ecully Cedex, France \\ ${ }^{\mathrm{b}}$ Department of Mechanical Engineering, University of Michigan, 3112 G. G. Brown, Ann Arbor, MI 48109-2125, USA
}

A new freq uercy-time domain procedure, the dynamic Lagrangian mixed freq uencytime method (DLFT), is proposed to calculate the non-linear steady state response to periodic excitation of structural systems subject to dry friction damping. In this formulation, the dynamic Lagrangians are defined as the non-linear contact forces obtained from the eq uations of motion in the freq uency domain, with the adjunction of a penalization on the difference between the interface displacements calculate by the nonlinear solver in the frequency domain and those calculated in the time domain from the non-linear contact forces, thus accounting for Coulomb friction and non-penetration conditions. The dynamic Lagrangians allow one to solve for the non-linear forces between two points in contact without using artifacts such as springs. The new DLFT method is thus particularly well suited to handling finite element models of structures in frictional contact, as it does not require a special model for the contact interface. Dynamic Lagrangians are also better suited to frequency-domain friction problems than the traditional time-domain method of augmented Lagrangians. Furthermore, a reduction of the non-linear system to relative interface displacements is introduced to decrease the computation time. The DLFT method is validated for a beam in contact with a flexible dry friction element connected to ground, for frictional constraints that feature two-dimensional relative motion. Results are also obtained for a large-scale structural system with a large number of one-dimensional dry-friction dampers. The DLFT method is shown to be accurate and fast, and it does not suffer from convergence problems, at least in the examples studied.

\footnotetext{
*Corresponding author. Tel. : +33-4-72-18-64-71; fax: +33-4-72-18-91-44.

E-mail addresses: nacivet@mecasola.ec-lyon.fr (S. Nacivet), pierre@umich.edu (C. Pierre), fabrice.thouverez@ec-lyon.fr (F. Thouverez).
} 


\section{Introduction}

In the field of turbomachinery, considerable efforts have been devoted to the formulation of predictive models to describe the non-linear behavior of rotor blades with dry friction dampers attached. While the vibration response of these systems can be obtained using a time integration of the equations of motion, this approach often carries a prohibitive cost, particularly for systems with small damping. Hence over the years various methods have been developed to calculate efficiently the steady state response of dry-friction-damped structural systems to periodic excitation.

Frequency-domain methods such as the harmonic balance method (HBM) allow for significant reduction in computational cost compared to time-domain numerical integration. The HBM and its variation, the incremental HBM, have been used extensively to predict the forced response of structural systems with attached friction dampers [1-3]. These studies have typically been conducted for a few simple friction dampers (with one-dimensional motion at the contact point) and have usually accounted for a single temporal harmonic of the motion. Some researchers have considered multi-harmonic response, but the HBM quickly becomes cumbersome, as it requires significant analytical work to capture transitions between sticking and slipping states accurately [1]. In 1989, Cameron and Griffin [4] pioneered the development of the alternating frequency/ time-domain (AFT) method, which circumvents this difficulty by evaluating the non-linear contact forces in the time domain. This way Coulomb conditions can be applied in a straightforward way, by estimating the stick-slip transitions at each iterative step. Nevertheless, all these methods (HBM, IHBM, AFT) may suffer convergence problems when a NewtonRaphson algorithm is used to solve the strongly non-linear frequency-domain equations, particularly for complex damper configurations (e.g., three-dimensional motion of the contact point). More recently, Guillen and Pierre [5] modified the AFT method by integrating exactly the non-linear contact forces in the time domain and using a robust non-linear Broyden solver. Their method was shown to produce rapid convergence for large-scale systems with large numbers of one-dimensional friction dampers. Finally, Poudou [6] achieved excellent convergence for complex damper models (e.g., with variable normal load and separation at the contact point) by using a hybrid Powell non-linear solver programmed by Garbow et al. [7]. The hybrid Powell algorithm was selected for use in the present work (see Ref. [8] for details of the Powell method).

For problems involving two structures with a common contact interface where dry friction occurs, a contact element is usually defined by a (set of) linear spring(s) and a (set of) friction element(s). Note that in turbomachinery, the two structures in contact may consist of a blade and a friction damper, or of two blades in the case of direct contact at a shroud, or of a blade and the disk in the case of a dovetail attachment. In most studies performed to date, the friction damper models considered have been simple enough that the damper itself can be included in the contact element, with the linear springs then representing the damper's flexibility, thereby eliminating the need to represent the damper as a separate structure. For instance, one-directional springs are routinely included in contact elements in order to capture the flexibility of one-dimensional dampers [9-13].

In recent years, however, friction dampers with complex motion at the contact interface have been considered, for example dampers featuring two- and three-dimensional motion with variable normal load and separation [6,14-19] and wedge dampers [20,21]. Also, researchers have studied 
the influence of microslip on the response of blade/damper systems, using continuous contact models that assume a contact shear layer with a given normal pressure profile (constant or parabolic) [22-24]. These methods rely on the judicious choice of microslip model parameters to agree with experimental data. In the context of the finite element method, Berger et al. [25] has proposed to improve the microslip description by defining an interpolation on the effective friction coefficient (the tangential to normal contact force ratio). Note that in finite element models, microslip can be studied using a set of contact elements on the interface. However, in the frequency domain, the springs included in the contact elements lead to a degradation of the interface model. Note that if the method of augmented Lagrangians [26-29] were used, the inclusion of springs in the contact elements would be unnecessary, and the finite element models of the two structures in frictional contact could be used to solve directly for the non-linear forces.

While the method of augmented Lagrangians is a popular one in time-domain formulations of contact problems, to date this type of approach has not been used for frequency-domain response predictions. In this paper, a new dynamic Lagrangian mixed frequency-time method (DLFT) is introduced to calculate the steady state forced response of dry-friction-damped systems. This approach is essentially based upon the augmented Lagrangians methodology, but adapted to a frequency-domain framework. However, it is important to note that our new DLFT method does not make specific use of augmented Lagrangians, although they could have been incorporated in the approach and can give good results. This is because in order to avoid prohibitive computation time, the augmented Lagrangians at a given frequency need to be updated using the non-linear contact forces at the previous frequency, hence requiring a fairly large penalty coefficient. This means that the range in which the penalty coefficient can be chosen to achieve good precision and avoid numerical stability problems is very limited, making the method somewhat impractical. Therefore, in order to ensure robust and fast convergence, dynamic Lagrangians, which are based explicitly on the equations of motion in the frequency domain and do not suffer updating problems are introduced.

In this paper, an elastic, homogeneous, and isotropic structure subject to periodic external excitation is considered, in which sets of points are in frictional contact. The structure is subject to small, periodic deformations around an equilibrium state. Also, gyroscopic effects are neglected and Coulomb's law is used to model dry friction, without considerations of wear mechanisms. In Section 2, the new DLFT algorithm is presented, along with the introduction of the dynamic Lagrangians. A reduction of the number of non-linear equations is also described, based on relative displacements of nodes at the interface. In Section 3, the DLFT method is validated for a beam attached to a two-dimensional friction damper with variable normal load, and numerical results are presented for a large-scale system with many one-dimensional friction dampers attached. Finally, conclusions are given in Section 4.

\section{Method of analysis}

A general elastic structure with $N$ degrees of freedom (d.o.f.) is considered. The linear structural model may be derived using the finite element method or any other method, such as component mode synthesis, but all degrees of freedom where friction takes place must be retained as physical 
co-ordinates. A contact element is defined as a set of two nodes between which frictional contact may occur. The nodes for all the contact elements in the structure are called the non-linear nodes, and the remaining nodes are referred to as the linear nodes. For such a structure, the equations of motion can be written as

$$
[\mathbf{M}]\{\ddot{\mathbf{U}}\}+[\mathbf{C}]\{\dot{\mathbf{U}}\}+[\mathbf{K}]\{\mathbf{U}\}+\left\{\mathbf{F}_{c}\right\}=\left\{\mathbf{F}_{e x}\right\},
$$

where the linear structure is defined by its mass matrix $[\mathbf{M}]$, stiffness matrix $[\mathbf{K}]$ and viscous damping matrix $[\mathbf{C}]$. The vectors $\{\mathbf{U}\},\{\dot{\mathbf{U}}\}$ and $\{\ddot{\mathbf{U}}\}$ are, respectively, the displacement, velocity, and acceleration of the structure, $\left\{\mathbf{F}_{e x}\right\}$ is the vector of the external forces (periodic excitation at frequency $\omega$ ), and $\left\{\mathbf{F}_{c}\right\}$ represents the non-linear contact forces due to friction. Herein, vectors are boldfaced with braces, and matrices are boldfaced with brackets.

Next, assuming steady state periodic response, these equations are transformed to the frequency domain. The Fourier series expansion of the displacement $\{\mathbf{U}\}$ is

$$
\{\mathbf{U}\}(t)=\sum_{k=0}^{N_{h}}\{\mathbf{U}\}_{k}^{c} \cos (k \omega t)+\sum_{k=1}^{N_{h}}\{\mathbf{U}\}_{k}^{s} \sin (k \omega t),
$$

where $N_{h}$ is the number of temporal harmonics retained.

The multi-harmonic displacement vector in the frequency domain is defined as:

$$
\{\tilde{\mathbf{U}}\}=\left\{\{\mathbf{U}\}_{0}^{c \mathrm{~T}}\{\mathbf{U}\}_{1}^{c \mathrm{~T}}\{\mathbf{U}\}_{1}^{s \mathrm{~T}} \ldots\{\mathbf{U}\}_{N_{h}}^{c \mathrm{~T}}\{\mathbf{U}\}_{N_{h}}^{s \mathrm{~T}}\right\}^{\mathrm{T}},
$$

where the tilde refers to a multi-harmonic frequency-domain vector. The multi-harmonic vectors for the non-linear contact forces, $\left\{\tilde{\mathbf{F}}_{c}\right\}$, and the external forcing, $\left\{\tilde{\mathbf{F}}_{e x}\right\}$, can be defined in the same way. Then, the equation of motion (1) can be written in the frequency domain as

$$
[\mathbf{\Lambda}]\{\tilde{\mathbf{U}}\}+\left\{\tilde{\mathbf{F}}_{c}\right\}=\left\{\tilde{\mathbf{F}}_{e x}\right\},
$$

where

$$
\begin{gathered}
{[\mathbf{\Lambda}]=\left[\begin{array}{cc}
{[\mathbf{K}]} & {[\mathbf{0}]} \\
{[\mathbf{0}]} & {\left[\boldsymbol{\Lambda}_{h}\right]}
\end{array}\right], \text { and }} \\
{\left[\boldsymbol{\Lambda}_{h}\right]_{k}=\left[\begin{array}{cc}
-(k \omega)^{2}[\mathbf{M}]+[\mathbf{K}] & k \omega[\mathbf{C}] \\
-k \omega[\mathbf{C}] & -(k \omega)^{2}[\mathbf{M}]+[\mathbf{K}]
\end{array}\right], \quad k=1, \ldots, N_{h}}
\end{gathered}
$$

where $\left[\boldsymbol{\Lambda}_{h}\right]$ is the block-diagonal dynamic stiffness matrix, whose $k$ th block $\left[\boldsymbol{\Lambda}_{h}\right]_{k}$ defines the dynamic stiffness matrix for the $k$ th harmonic. Since the contact force is null for a linear node, Eq. (4) can be organized according to the linear (subscript $l n$ ) and non-linear (subscript $n l$ ) node displacements, as

$$
\left[\begin{array}{ll}
{[\boldsymbol{\Lambda}]_{l n, l n}} & {[\boldsymbol{\Lambda}]_{l n, n l}} \\
{[\mathbf{\Lambda}]_{n l, l n}} & {[\boldsymbol{\Lambda}]_{n l, n l}}
\end{array}\right]\left\{\begin{array}{c}
\{\tilde{\mathbf{U}}\}_{l n} \\
\{\tilde{\mathbf{U}}\}_{n l}
\end{array}\right\}+\left\{\begin{array}{c}
\left\{\tilde{\mathbf{0}}_{\}_{l n}}\right. \\
\left\{\tilde{\mathbf{F}}_{c}\right\}_{n l}
\end{array}\right\}=\left\{\begin{array}{c}
\left\{\tilde{\mathbf{F}}_{e x}\right\}_{l n} \\
\left\{\tilde{\mathbf{f}}_{e x}\right\}_{n l}
\end{array}\right\} .
$$

Note that $\left\{\tilde{\mathbf{F}}_{c}\right\}_{\ln }$ is null by definition.

Eq. (5) can be reduced to the non-linear d.o.f.'s only as

$$
\boldsymbol{\Lambda}_{\text {red }}\{\tilde{\mathbf{U}}\}_{n l}+\left\{\tilde{\mathbf{F}}_{c}\right\}_{n l}=\left\{\tilde{\mathbf{F}}_{r e d}\right\},
$$


where $\left[\boldsymbol{\Lambda}_{\text {red }}\right]$ and $\left\{\tilde{\mathbf{F}}_{\text {red }}\right\}$ are, respectively, the reduced dynamic stiffness matrix and the reduced external force vector:

$$
\begin{aligned}
{\left[\boldsymbol{\Lambda}_{r e d}\right] } & =[\boldsymbol{\Lambda}]_{n l, n l}-[\boldsymbol{\Lambda}]_{n l, l n}[\mathbf{\Lambda}]_{l n, l n}^{-1}[\mathbf{\Lambda}]_{l n, n l}, \\
\left\{\tilde{\mathbf{F}}_{r e d}\right\} & =\left\{\tilde{\mathbf{F}}_{e x}\right\}_{n l}-[\boldsymbol{\Lambda}]_{n l, l n}[\mathbf{\Lambda}]_{l n, l n}^{-1}\left\{\tilde{\mathbf{F}}_{e x}\right\}_{l n} .
\end{aligned}
$$

Eq. (6) is a set of $N_{n l}\left(2 N_{h}+1\right)$ non-linear equations, where $N_{n l}$ is the number of non-linear d.o.f.'s corresponding to the non-linear nodes (i.e., the contact d.o.f.'s).

Once the displacements of the non-linear d.o.f.'s, $\{\tilde{\mathbf{U}}\}_{n l}$, are known, $\{\tilde{\mathbf{U}}\}_{l n}$ can be simply solved as

$$
\{\tilde{\mathbf{U}}\}_{l n}=[\boldsymbol{\Lambda}]_{l n, l n}^{-1}\left(\left\{\tilde{\mathbf{F}}_{e x}\right\}_{l n}-[\boldsymbol{\Lambda}]_{l n, n l}\{\tilde{\mathbf{U}}\}_{n l}\right) .
$$

Because the computational time required increases rapidly with the number of non-linear unknowns, a secondary reduction is proposed to divide by two the size of the non-linear problem.

For each contact element defined previously as a set of two nodes, one node is selected arbitrarily to be a reference in the observation of the motion of the other node. Thus, the relative displacement can be introduced as (see Fig. 1):

$$
\left\{\tilde{\mathbf{U}}_{r}\right\}=\{\tilde{\mathbf{U}}\}_{n l, o b s}-\{\tilde{\mathbf{U}}\}_{n l, r e f},
$$

where the subscripts $r e f$ and $o b s$ refer to the reference and the observed nodes, respectively, and the $i$ th elements of these vectors correspond to the $i$ th contact element.

In addition, the inverse matrix of $\left[\boldsymbol{\Lambda}_{r e d}\right]$ is defined as

$$
[\mathbf{S}]=\left[\boldsymbol{\Lambda}_{r e d}\right]^{-1}
$$

and the multi-harmonic vector of Lagrange multipliers $\{\tilde{\boldsymbol{\lambda}}\}$ as

$$
\{\tilde{\lambda}\}=\left\{\tilde{\mathbf{F}}_{c}\right\}_{n l, o b s}=-\left\{\tilde{\mathbf{F}}_{c}\right\}_{n l, r e f} .
$$

The Lagrange multipliers can be described as the tangential contact forces caused by friction and the normal contact forces that ensure there is no interpenetration.

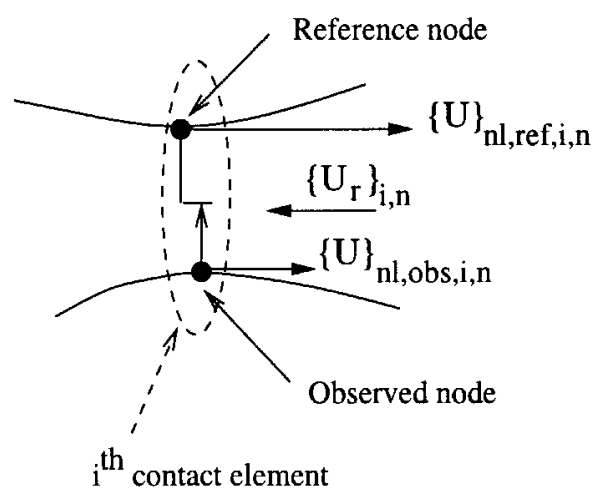

Fig. 1. Relative displacement representation for the $i$ th contact element. 
Using Eqs. (6), (11), and (12), the displacement vector $\{\tilde{\mathbf{U}}\}_{n l}$ is organized according to the reference and the observed nodes, as

$$
\left\{\begin{array}{l}
\{\tilde{\mathbf{U}}\}_{n l, o b s} \\
\{\tilde{\mathbf{U}}\}_{n l, r e f}
\end{array}\right\}=\left[\begin{array}{ll}
{[\mathbf{S}]_{\text {obs,obs }}} & {[\mathbf{S}]_{\text {obs }, r e f}} \\
{[\mathbf{S}]_{\text {ref,obs }}} & {[\mathbf{S}]_{\text {ref }, \text { ref }}}
\end{array}\right]\left\{\begin{array}{c}
\left\{\tilde{\mathbf{F}}_{\text {red }}\right\}_{\text {obs }}-\{\tilde{\boldsymbol{\lambda}}\} \\
\left\{\tilde{\mathbf{F}}_{\text {red }}\right\}_{\text {ref }}+\{\tilde{\boldsymbol{\lambda}}\}
\end{array}\right\} .
$$

Using Eqs. (10) and (13), a reduced equation of motion in the relative displacements of the observed nodes of the contact elements can be obtained in the frequency domain, as

$$
\left[\boldsymbol{\Lambda}_{r}\right]\left\{\tilde{\mathbf{U}}_{r}\right\}+\{\tilde{\boldsymbol{\lambda}}\}=\left\{\tilde{\mathbf{F}}_{r}\right\},
$$

where $\left[\boldsymbol{\Lambda}_{r}\right]=\left[[\mathbf{S}]_{r e f, r e f}+[\mathbf{S}]_{o b s, o b s}-[\mathbf{S}]_{r e f, o b s}-[\mathbf{S}]_{o b s, r e f}\right]^{-1}$ and $\left\{\tilde{\mathbf{F}}_{r}\right\}=\left[\boldsymbol{\Lambda}_{r}\right]\left(\left([\mathbf{S}]_{o b s, o b s}-[\mathbf{S}]_{o b s, r e f}\right)\right.$ $\left.\left\{\tilde{\mathbf{F}}_{\text {red }}\right\}_{\text {obs }}-\left([\mathbf{S}]_{\text {ref }, \text { ref }}-[\mathbf{S}]_{\text {ref,obs }}\right)\left\{\tilde{\mathbf{F}}_{\text {red }}\right\}_{\text {ref }}\right)$.

The subscript $r$ refers to the system reduced to the relative displacements of the observed nodes.

In order to solve Eq. (14), a non-linear solver based on the hybrid Powell algorithm [8] is used to find the zeros of the non-linear vector function:

$$
\mathbf{f}\left(\left\{\tilde{\mathbf{U}}_{r}\right\}\right)=\left[\boldsymbol{\Lambda}_{r}\right]\left\{\tilde{\mathbf{U}}_{r}\right\}+\{\tilde{\lambda}\}-\left\{\tilde{\mathbf{F}}_{r}\right\} .
$$

Once $\left\{\tilde{\mathbf{U}}_{r}\right\}$ is solved for, $\{\tilde{\boldsymbol{\lambda}}\}$ is known, and then $\{\tilde{\mathbf{U}}\}_{n l}$ can be obtained from Eq. (13). A flowchart of the dynamic Lagrangian mixed frequency-time (DLFT) algorithm is shown in Fig. 2.

It is difficult to develop a method in which non-linear forces are handled in the frequency domain, because a large number of temporal harmonics are required to evaluate them accurately. Therefore, non-linear contact and friction forces are generally calculated using a time-marching procedure in the time domain, with criteria for the states of sticking, slipping, separation, and non-penetration. In the DLFT method developed here, this necessary time-marching procedure is implemented for the non-linear contact forces via the introduction of a "dynamic Lagrangian" formulation, which relies on penalizing the difference between the relative interface displacements obtained from the non-linear solver in the frequency domain, $\left\{\tilde{\mathbf{U}}_{r}\right\}$, and those calculated in the

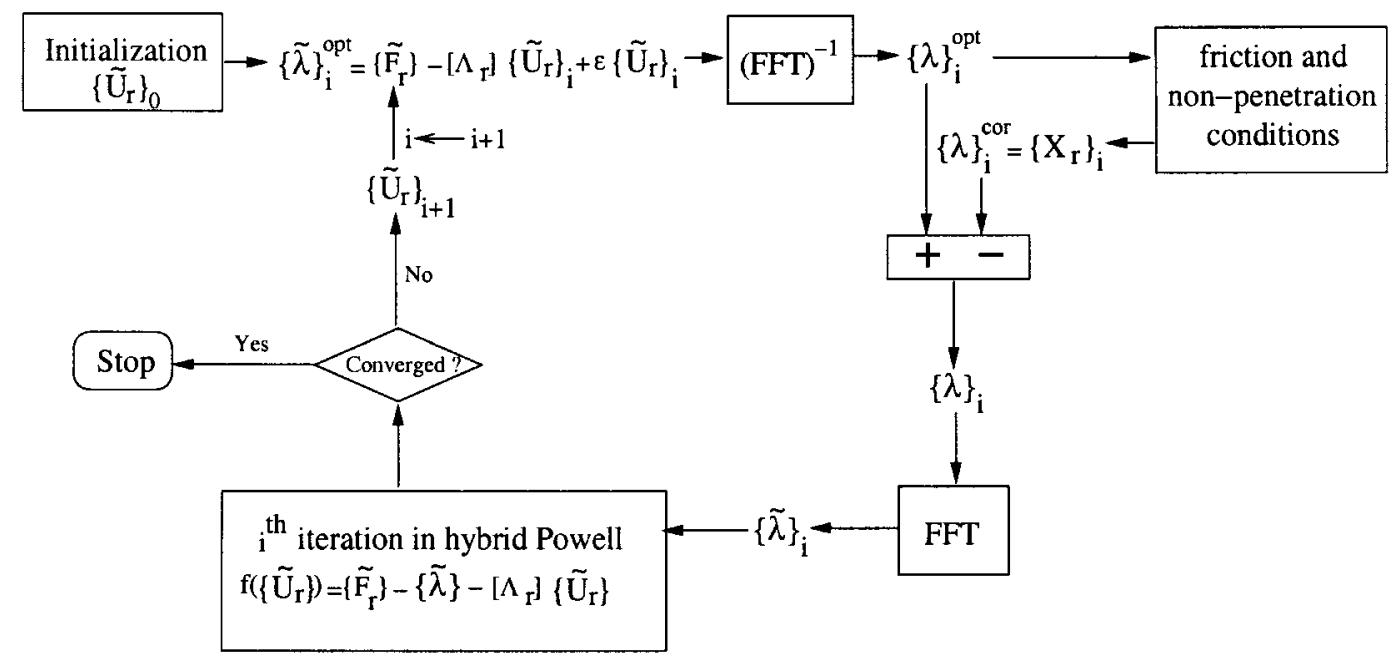

Fig. 2. Implementation of the DLFT. 
time domain from the non-linear contact forces evaluated from $\left\{\tilde{\mathbf{U}}_{r}\right\}$, checking for Coulomb friction and non-penetration conditions. This vector of relative displacements calculated in the time domain will be referred to as $\left\{\mathbf{X}_{r}\right\}$, and the corresponding Fourier transformed vector as $\left\{\tilde{\mathbf{X}}_{r}\right\}$. Note that in traditional formulations, the corrections applied to non-linear force calculations depend on the hypothesis made for the states of the contact elements. In the DLFT method, however, the states of the contact elements result from the applied corrections, hence constituting an indirect approach to define these states. When convergence is reached, Eq. (14) is satisfied and one then has

$$
\left\{\tilde{\mathbf{X}}_{r}\right\}=\left\{\tilde{\mathbf{U}}_{r}\right\} .
$$

This means that the interface displacements obtained from the non-linear solver then match those integrated in the time domain using the friction and non-penetration conditions.

At each iteration, the non-linear solution algorithm require the evaluation of the function $\mathbf{f}\left(\left\{\tilde{\mathbf{U}}_{r}\right\}\right)$, which itself calls for the evaluation of $\{\tilde{\boldsymbol{\lambda}}\}$. Using Eqs. (14) and (16), a new formulation for $\{\tilde{\boldsymbol{\lambda}}\}$, is proposed, in which the dynamic Lagrangians is defined as

$$
\{\tilde{\boldsymbol{\lambda}}\}=\left\{\tilde{\mathbf{F}}_{r}\right\}-\left[\boldsymbol{\Lambda}_{r}\right]\left\{\tilde{\mathbf{U}}_{r}\right\}+\varepsilon\left(\left\{\tilde{\mathbf{U}}_{r}\right\}-\left\{\tilde{\mathbf{X}}_{r}\right\}\right),
$$

where $\varepsilon$ is a penalty coefficient chosen arbitrarily as positive, which influences the speed of convergence.

Note that if the reduction to the relative interface displacements were not performed, the dynamic Lagrangians could still be defined using the equations of motion for the non-linear displacements in Eq. (6). Also note that Eq. (17) ensures that if one of Eqs. (14) or (16) is verified, then the other one is satisfied as well. There are several benefits to the use of dynamic Lagrangians: first, they do not require consideration of the non-linear contact forces as unknowns, as Lagrange multipliers do; second, the dynamic Lagrangians formulation is better suited than an augmented Lagrangians formulation in the frequency domain, because there is no problem with their update; third, the penalty coefficient for dynamic Lagrangians can be chosen to be smaller than for augmented Lagrangians, which allows for faster convergence.

In order to compute $\{\tilde{\lambda}\}$ at a given iteration, one needs to determine $\left\{\tilde{\mathbf{X}}_{r}\right\}$, while $\left\{\tilde{\mathbf{U}}_{r}\right\}$ is provided by the non-linear solver. Now separate the expression for $\{\tilde{\lambda}\}$, Eq. (17), into two parts. The first one, $\{\tilde{\lambda}\}^{o p t}$, will be referred to as the optimization force vector:

$$
\{\tilde{\boldsymbol{\lambda}}\}^{o p t}=\left\{\tilde{\mathbf{F}}_{r}\right\}-\left[\boldsymbol{\Lambda}_{r}\right]\left\{\tilde{\mathbf{U}}_{r}\right\}+\varepsilon\left\{\tilde{\mathbf{U}}_{r}\right\} .
$$

Note that $\{\tilde{\lambda}\}^{\text {opt }}$ can be evaluated in the frequency domain because $\left\{\tilde{\mathbf{U}}_{r}\right\}$ is provided by the hybrid Powell solver. The second part, $\{\tilde{\boldsymbol{\lambda}}\}^{\text {cor }}$, will be referred to as the non-linear corrective force vector:

$$
\{\tilde{\boldsymbol{\lambda}}\}^{\mathrm{cor}}=\varepsilon\left\{\tilde{\mathbf{X}}_{r}\right\} .
$$

Note that $\{\tilde{\boldsymbol{\lambda}}\}^{\text {cor }}$ is unknown because $\left\{\tilde{\mathbf{X}}_{r}\right\}$ is undefined at this stage. Using Eqs. (17)-(19), $\{\tilde{\boldsymbol{\lambda}}\}$ can be rewritten as

$$
\{\tilde{\lambda}\}=\{\tilde{\lambda}\}^{o p t}-\{\tilde{\lambda}\}^{c o r} .
$$


Performing an inverse Fourier transform, $\{\lambda\}$ can be expressed in the time domain. Eq. (20) becomes

$$
\{\boldsymbol{\lambda}\}_{n}=\{\boldsymbol{\lambda}\}_{n}^{\text {opt }}-\{\boldsymbol{\lambda}\}_{n}^{\mathrm{cor}},
$$

where the subscript $n$ denotes the $n$th time step $t_{n}$.

Next, $\{\lambda\}_{n}^{c o r}$ is calculated simply by using the Coulomb friction and non-penetration conditions. It is important to note that while $\left\{\mathbf{X}_{r}\right\}_{n}$ allows one to justify the theoretical approach, it is not necessary to calculate it in the case of node-to-node contact elements. The calculation of $\{\lambda\}^{\text {cor }}$, which is proportional to $\left\{\mathbf{X}_{r}\right\}$, is sufficient. An iterative formulation for the corrective force $\{\boldsymbol{\lambda}\}_{n}^{\text {cor }}$ is defined in the time domain as:

$$
\{\lambda\}_{n}^{c o r}=\{\lambda\}_{n-1}^{c o r}+\Delta_{n}\{\lambda\}^{c o r} .
$$

Here $\Delta_{n}\{\lambda\}^{\text {cor }}$ is the correction applied on $\{\lambda\}_{n}^{\text {opt }}-\{\lambda\}_{n-1}^{\text {cor }}$ in order to take into account the constraint conditions at the interfaces, where $\Delta_{n}$ is an operator of finite difference between the $n$th and $(n-1)$ th time steps.

The initialization of $\{\lambda\}_{n=-1}^{c o r}$ can be chosen as

$$
\{\lambda\}_{n=-1}^{c o r}=0 .
$$

Next, $\Delta_{n}\{\lambda\}_{i}^{c o r}$ is expressed for the $i$ th contact element in order to ensure non-penetration and satisfy Coulomb friction conditions. In the case of separation of the interfaces, one simply has to impose $\{\lambda\}_{i, n}^{\text {cor }}=\{\lambda\}_{i, n}^{\text {opt } t}$ to obtain $\{\boldsymbol{\lambda}\}_{i, n}=0$. Then $\Delta_{n}\{\boldsymbol{\lambda}\}_{i}^{\text {cor }}$ is given by

$$
\Delta_{n}\{\lambda\}_{i}^{\text {cor }}=\{\lambda\}_{i, n}^{\text {opt }}-\{\lambda\}_{i, n-1}^{\text {opt }} \text {. }
$$

In the case of sticking interfaces, the condition is also very simple, namely

$$
\Delta_{n}\{\boldsymbol{\lambda}\}_{i}^{c o r}=0 .
$$

Using Eq. (19), it is easily deduced that $\Delta_{n}\left\{\mathbf{X}_{r}\right\}_{i}=0$.

In the case of sliding interfaces, some additional derivations are necessary, as follows. In the normal direction, there still exists the sticking condition:

$$
\Delta_{n}\{\lambda\}_{N, i}^{c o r}=0,
$$

where the subscript $N$ indicates the normal direction. However, in the tangential direction, one needs to express the contact force as

$$
\{\lambda\}_{T, i, n}=\{\lambda\}_{T, i, n}^{o p t}-\{\lambda\}_{T, i, n-1}^{c o r}-\Delta_{n}\{\lambda\}_{T, i}^{c o r},
$$

where the subscript $T$ indicates the tangential plane (2-D) or direction (1-D).

The quantity $\Delta_{n}\{\lambda\}_{T, i}^{c o r}$ must be chosen such that the tangential contact force, $\{\lambda\}_{T, i, n}$, has the same orientation as the relative velocity, $\left\{\mathbf{W}_{r}\right\}_{n}$, and has the magnitude $\mu \mid\{\lambda\}_{N, i, n}$, where $\mu$ is the coefficient of friction. An approximation of $\left\{\mathbf{W}_{r}\right\}_{n}$ can be obtained by finite difference as

$$
\left\{\mathbf{W}_{r}\right\}_{n}=\frac{\left\{\mathbf{X}_{r}\right\}_{n}-\left\{\mathbf{X}_{r}\right\}_{n-1}}{\Delta_{n} t} .
$$

Using Eqs. (19) and (22), the relative velocity $\left\{\mathbf{W}_{r}\right\}_{n}$ can be expressed as

$$
\left\{\mathbf{W}_{r}\right\}_{n}=\frac{\Delta_{n}\{\lambda\}_{T}^{c o r}}{\varepsilon \Delta_{n} t} .
$$


When the contact element $i$ is undergoing slipping, the basic formulation for the tangential nonlinear forces is given by

$$
\{\lambda\}_{T, i, n}=\mu\left|\lambda_{N, i, n}\right| \frac{\left\{\mathbf{W}_{\mathbf{r}}\right\}_{i, n}}{\left\|\left\{\mathbf{W}_{\mathbf{r}}\right\}_{i, n}\right\|},
$$

where $\|$.$\| is the Euclidian norm. This means that the tangential force for the contact element i$ is aligned with the relative velocity, $\left\{\mathbf{W}_{r}\right\}_{i, n}$, because the tangential force, $\{\boldsymbol{\lambda}\}_{T, i, n}$, is defined as the opposite of the frictional force. Using Eq. (29), Eq. (30) can be rewritten as

$$
\{\lambda\}_{T, i, n}=\mu\left|\lambda_{N, i, n}\right| \frac{\Delta_{n}\{\lambda\}_{T, i}^{c o r}}{\left\|\Delta_{n}\{\lambda\}_{T, i}^{c o r}\right\|} .
$$

Substituting Eq. (31) into Eq. (27), one deduces $\Delta_{n}\{\lambda\}_{T, i}^{\text {cor }}$ as

$$
\Delta_{n}\{\lambda\}_{T, i}^{c o r}=\frac{\left\|\Delta_{n}\{\lambda\}_{T, i}^{c o r}\right\|}{\left\|\Delta_{n}\{\boldsymbol{\lambda}\}_{T, i}^{c o r}\right\|+\mu\left|\lambda_{N, i, n}\right|}\left(\{\lambda\}_{T, i, n}^{o p t}-\{\lambda\}_{T, i, n-1}^{c o r}\right) .
$$

Then, $\Delta_{n}\{\boldsymbol{\lambda}\}_{T, i}^{\text {cor }}$ has the same orientation as $\{\boldsymbol{\lambda}\}_{T, i, n}^{\text {opt }}-\{\boldsymbol{\lambda}\}_{T, i, n-1}^{\text {cor }}$.

The norm of $\Delta_{n}\{\lambda\}_{T, i}^{c o r}$ is obtained simply using the norm of Eq. (32):

$$
\left\|\Delta_{n}\{\boldsymbol{\lambda}\}_{T, i}^{c o r}\right\|=\left\|\{\boldsymbol{\lambda}\}_{T, i, n}^{o p t}-\{\boldsymbol{\lambda}\}_{T, i, n-1}^{c o r}\right\|-\mu\left|\lambda_{N, i, n}\right| .
$$

Using last two equations, one can calculate the norm and the orientation of $\Delta_{n}\{\lambda\}_{T, i}^{c o r}$.

Consequently, one can able to calculate $\{\lambda\}_{n}^{\text {cor }}$ using Eq. (21) and $\{\lambda\}_{n}$ using Eq. (22).

The above correction procedure is depicted in Fig. 3 for the case of a 2-D relative motion.

Next, the conditions of transition between the different states (stick, slip, separation) must be defined. For the transition between contact and separation, a test on the sign of the normal contact force, $\lambda_{N, i, n}$, is sufficient. The normal orientation of the contact element is important in this case.

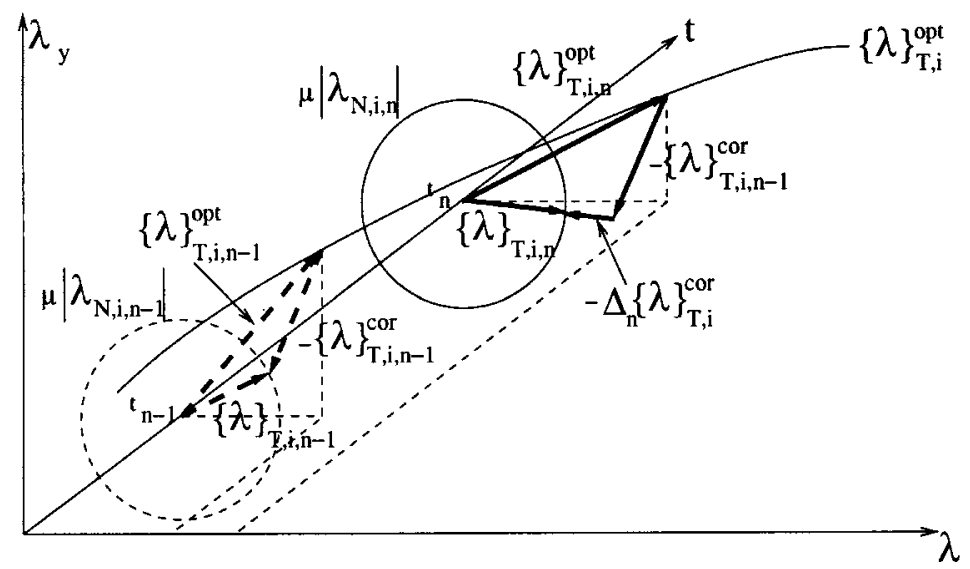

Fig. 3. Schematic of the correction procedure for the tangential force at time steps $n-1$ and $n$. 
For the transition between sticking and slipping, one notes that $\Delta_{n}\{\lambda\}_{T, i, n}^{\text {cor }}$ verifies

$$
\begin{array}{ll}
\Delta_{n}\{\boldsymbol{\lambda}\}_{T, i, n}^{c o r}=\{\boldsymbol{0}\} & \text { if }\left\|\{\boldsymbol{\lambda}\}_{T, i, n}^{o p t}-\{\boldsymbol{\lambda}\}_{T, i, n-1}^{c o r}\right\|<\mu\left|\lambda_{N, i, n}\right|, \\
\Delta_{n}\{\boldsymbol{\lambda}\}_{T, i, n}^{c o r} \neq\{\mathbf{0}\} & \text { if }\left\|\{\boldsymbol{\lambda}\}_{T, i, n}^{o p t}-\{\boldsymbol{\lambda}\}_{T, i, n-1}^{c o r}\right\|>\mu\left|\lambda_{N, i, n}\right| .
\end{array}
$$

According to Eq. (19), it follows naturally that

$$
\begin{array}{ll}
\Delta_{n}\{\boldsymbol{\lambda}\}_{T, i, n}^{c o r}=\{\boldsymbol{0}\} & \text { if the } i \text { th contact element is sticking, } \\
\Delta_{n}\{\boldsymbol{\lambda}\}_{T, i, n}^{c o r} \neq\{\boldsymbol{0}\} & \text { if the } i \text { th contact element is slipping. }
\end{array}
$$

Then, the transitions are deduced from the violation of the following conditions:

$$
\begin{array}{ll}
\left\|\{\boldsymbol{\lambda}\}_{T, i, n}^{o p t}-\{\boldsymbol{\lambda}\}_{T, i, n-1}^{\mathrm{cor}}\right\|<\mu\left|\lambda_{N, i, n}\right| & \text { if the } i \text { th contact element is sticking, } \\
\left\|\{\boldsymbol{\lambda}\}_{T, i, n}^{o p t}-\{\boldsymbol{\lambda}\}_{T, i, n-1}^{c o r}\right\|>\mu\left|\lambda_{N, i, n}\right| & \text { if the } i \text { th contact element is slipping. }
\end{array}
$$

\section{Results}

In order to evaluate the validity and accuracy of the DLFT method, three numerical examples are considered. In the first two examples, the DLFT results are compared with those of a time integration performed with the commercial software ABAQUS [30] using the Lagrange multipliers method. The system considered is a beam with an attached flexible friction damper, constrained to have one-dimensional relative motion in the first case and two-dimensional relative motion in the second case. In the third example, results are obtained with the DLFT method for a large-scale system with a large number of beams, each being attached to a one-dimensional flexible friction damper.

The first system considered is a steel beam with a flexible dry-friction damper attached at three tenths of its length, as represented in Fig. 4a. The only retained d.o.f.'s are in the $X$ and $Z$ directions.

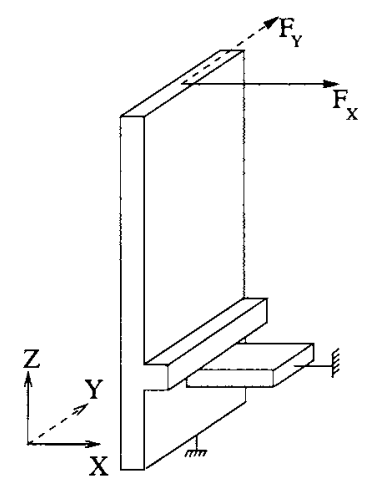

(a)

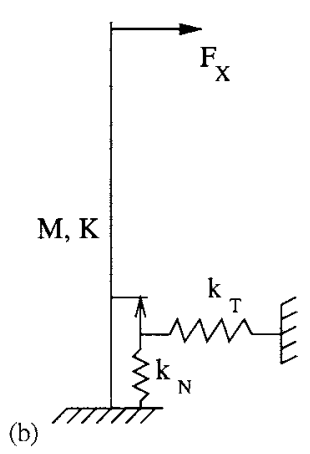

(b)

Fig. 4. (a) Geometry of the beam and damper system, (b) sketch of the dry friction damped beam, for a 2-D flexible damper model with mono-directional stiffnesses in both tangential and normal directions. 
The cross-section of the beam in the $X-Y$ plane is $0.01 \mathrm{~m} \times 0.1 \mathrm{~m}$ and its length is $0.5 \mathrm{~m}$. The platform where the damper is attached has the same cross-section in the $Z-Y$ plane as the beam in the $X-Y$ plane, but its length in $X$ direction is only $1 \mathrm{~mm}$. The finite element model of the beam consists of B21 beam elements with rectangular cross-section in ABAQUS [30]. This model is condensed out using Craig-Bampton component mode synthesis [31], retaining four active d.o.f.'s and the lowest two modes of free vibration of the beam fixed at the active d.o.f.'s. The four active d.o.f.'s correspond to two retained nodes (d.o.f.'s in $X$ and $Z$ directions for each node), the first one at the damper location and the second one at the free tip of the beam. A contact element is defined between the beam and the flexible damper, where the first d.o.f. corresponds to the tangential direction and the second to the normal direction. The coefficient of friction is $\mu=0.1$ and the viscous damping $\xi=0.01$. The flexible damper is defined by two mono-directional stiffnesses: $k_{T}=2.4 \times 10^{7} \mathrm{~N} / \mathrm{m}$ in the $X$ direction and $k_{N}=2.4 \times 10^{3} \mathrm{~N} / \mathrm{m}$ in the $Z$ direction. A pre-load of $1500 \mathrm{~N}$ is applied to the damper in the $Z$ direction. Notice that the normal d.o.f.'s are retained as unknowns. Although the displacements of the normal d.o.f.'s are small, they are required in the contact element definition of ABAQUS. Finally, a periodic excitation $F_{X}=50 \sin (\omega t)$ is applied at the free end of the beam (see Fig. 4b).

The time responses of the beam and the damper at the frequency $\omega=124 \mathrm{~Hz}$ are shown in Fig. 5a using the DLFT and time integration. Excellent agreement between the two methods is observed. The beam frequency response depicted in Fig. 5b shows a similar agreement at additional frequencies. For these calculations, 21 temporal harmonics of the response were retained in the DLFT method, which seemed sufficient to represent the response accurately.

However, it is important to notice that there is no systematic way of selecting a priori the appropriate number of harmonics. No convergence problem was encountered for either the DLFT or the time integration.
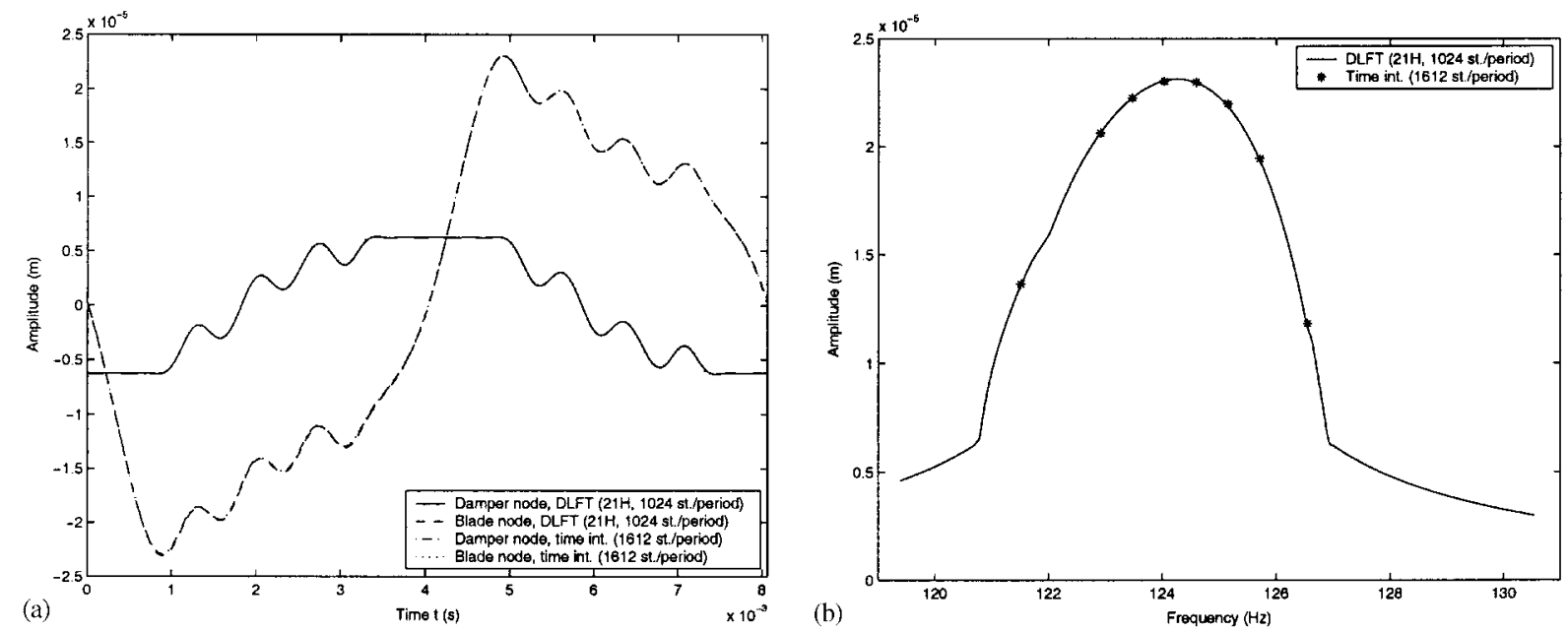

Fig. 5. (a) Beam displacement at the damper location, and damper displacement, compared using the DLFT method and time integration $\left(\xi=0.01, \mu=0.1, \omega=124 \mathrm{~Hz}, F_{X}=50 \sin (\omega t), N_{h}=21\right.$ and $\left.\varepsilon=8 \times 10^{7}\right)$. (b) Beam frequency response at the damper location $\left(\xi=0.01, \mu=0.1, F_{X}=50 \sin (\omega t), N_{h}=21\right.$ and $\left.\varepsilon=8 \times 10^{7}\right)$. 
It is difficult to compare the computational times of the DLFT and time-integration methods because there is no test of convergence on the steady state response in ABAQUS. However, an approximate comparison was made at the frequency $\omega=124 \mathrm{~Hz}$, in the case where the DLFT is performed with a zero initial solution at that frequency. The initial conditions were taken equal to zero as well for the ABAQUS time integration, which was carried out over 30 periods in order to obtain a sufficiently accurate steady state response. In this case, the time-integration method needed $342 \mathrm{~s}$ compared to $0.45 \mathrm{~s}$ for the DLFT. In addition, for this calculation at a single frequency, the DLFT method required 260 evaluations of the Jacobian matrix in the non-linear solver. If the same calculation were realized over a wide frequency range, the DLFT would need only two evaluations of the Jacobian at each frequency, thus reducing the time of calculation to $0.042 \mathrm{~s}$ per frequency.

The beam depicted in Fig. 4a is used in the second example as well. The finite element model of the beam consists of B31 beam elements with rectangular cross-section in ABAQUS [30]. This model is condensed out using Craig-Bampton component mode synthesis, now retaining six active d.o.f.'s and the lowest three modes of free vibration of the beam fixed at the active d.o.f.'s. The same two nodes as in the first example are retained, with three d.o.f.'s at each node. A stiffness element is added to model the flexible damper in the $Y$ direction. The value of this stiffness is $2.4 \times 10^{7} \mathrm{~N} / \mathrm{m}$, which is the same as that in the $X$ direction. A comparison of the DLFT and the time integration methods was realized at the frequency $\omega=124 \mathrm{~Hz}$ for two cases of excitation, using 21 harmonics in the DLFT computation.

In Fig. 6, both the displacements of the blade at the damper location and that of the damper are compared in the $X-Y$ plane with the time integration results, for a nearly rectilinear excitation: $F_{X}=50 \sin (\omega t)$ and $F_{Y}=500 \sin (\omega t)$.

The blade and damper time responses are given in Fig. 7 for the $X$ direction and Fig. 8 for the $Y$ direction. Additional frequencies were checked, as shown in Fig. 9.

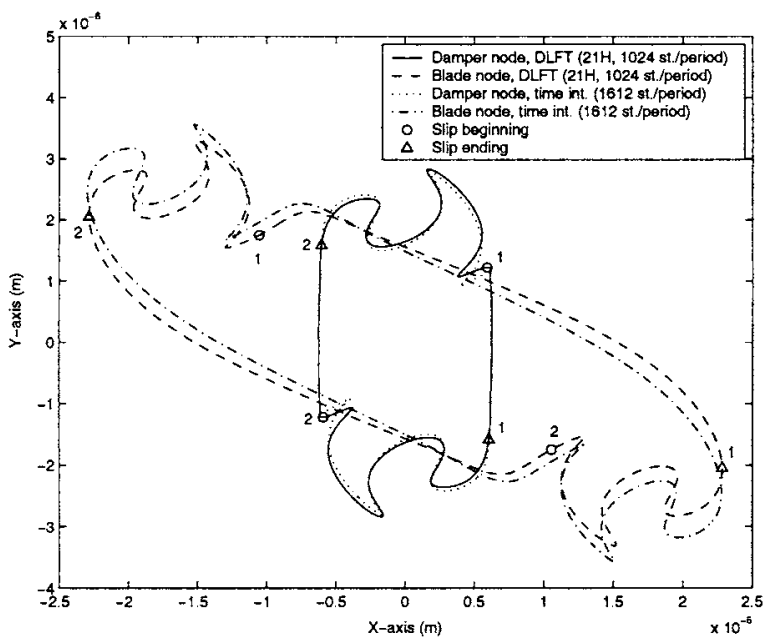

Fig. 6. Blade motion at the damper location, and damper motion, in the tangential plane, for case of a rectilinear excitation $\left(\xi=0.01, \mu=0.1, \omega=124 \mathrm{~Hz}, F_{X}=50 \sin (\omega t), F_{Y}=500 \sin (\omega t), N_{h}=21\right.$ and $\left.\varepsilon=8 \times 10^{7}\right)$. 


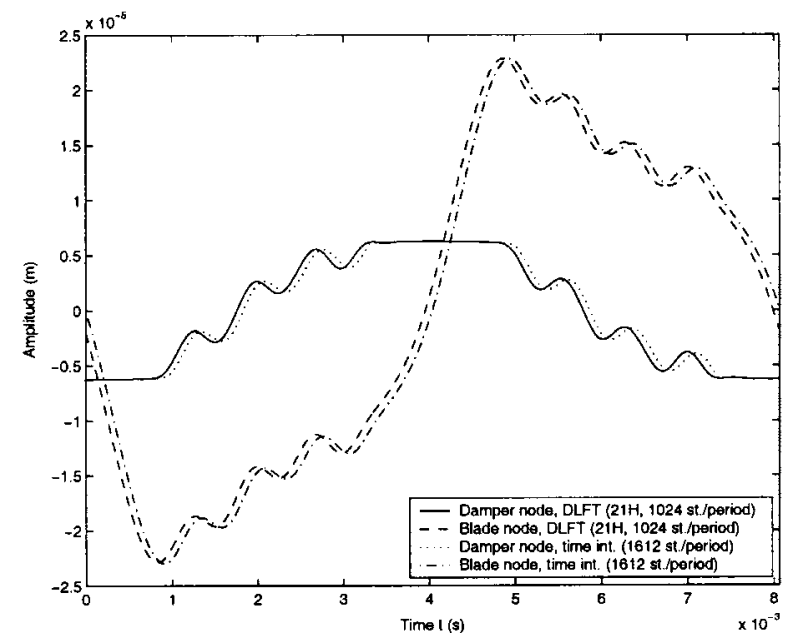

Fig. 7. Beam displacement at the damper location, and damper displacement, compared in the $X$ direction using the new DLFT method and time integration, for case of a rectilinear excitation $\left(\xi=0.01, \mu=0.1, \omega=124 \mathrm{~Hz}, F_{X}=\right.$ $50 \sin (\omega t), F_{Y}=500 \sin (\omega t), N_{h}=21$ and $\left.\varepsilon=8 \times 10^{7}\right)$.

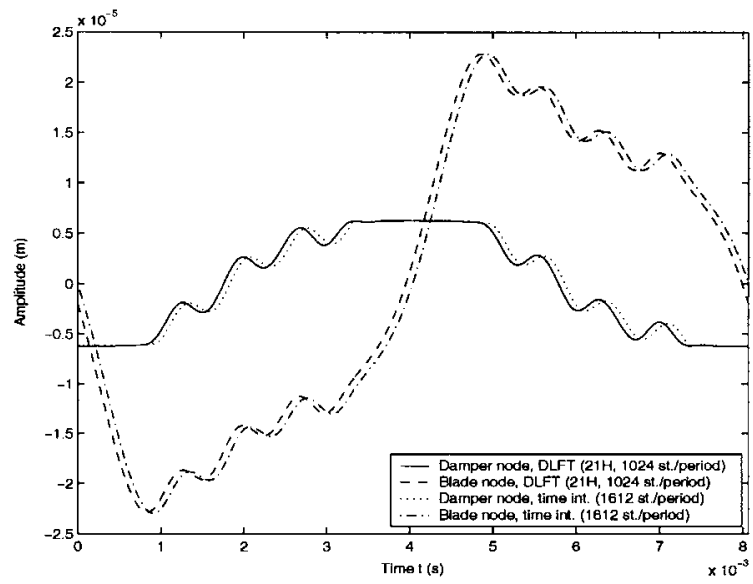

Fig. 8. Beam displacement at the damper location, and damper displacement, compared in the $Y$ direction using the new DLFT method and time integration, for case of a rectilinear excitation $\left(\xi=0.01, \mu=0.1, \omega=124 \mathrm{~Hz}, F_{X}=\right.$ $50 \sin (\omega t), F_{Y}=500 \sin (\omega t), N_{h}=21$ and $\left.\varepsilon=8 \times 10^{7}\right)$.

In Fig. 10, the comparison is repeated in the $X-Y$ plane for a circular excitation: $F_{X}=50 \sin (\omega t)$ and $F_{Y}=50 \cos (\omega t)$.

The blade and damper time responses are given in Fig. 11 for the $X$ direction and Fig. 12 for the $Y$ direction. Additional frequencies were checked, as shown in Fig. 13.

The displacements in the $\mathrm{Z}$ direction are not shown because they are small and are not of high interest. Of course, there is no separation.

The agreement of the DLFT with the time integration is good even though small differences may be noted due to numerical precision. The comparison in the time domain between the DLFT results and the time integration shows that there are small errors in amplitude (especially in the $Y$ 


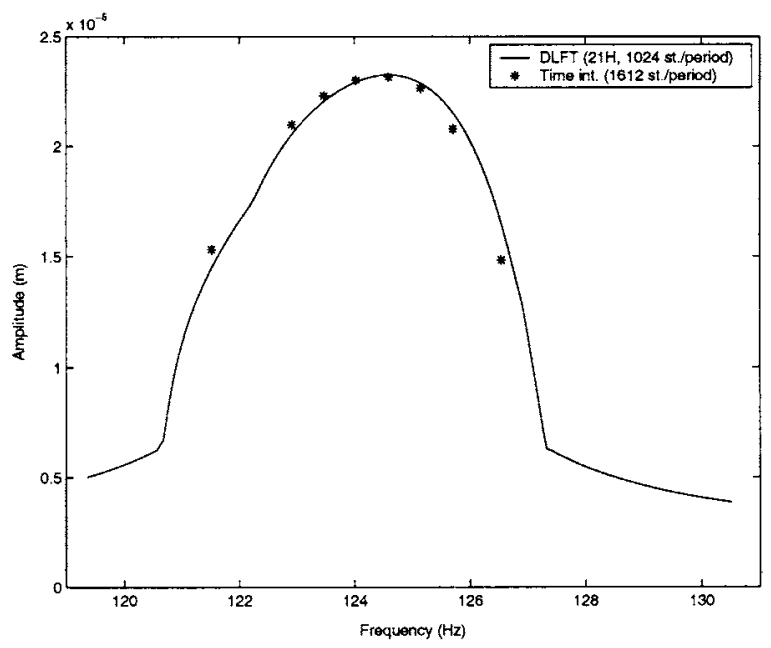

Fig. 9. Amplitude of the beam at the damper location for case of a rectilinear excitation $(\xi=0.01, \mu=0.1, \omega=$ $124 \mathrm{~Hz}, F_{X}=50 \sin (\omega t), F_{Y}=500 \sin (\omega t), N_{h}=21$ and $\left.\varepsilon=8 \times 10^{7}\right)$.

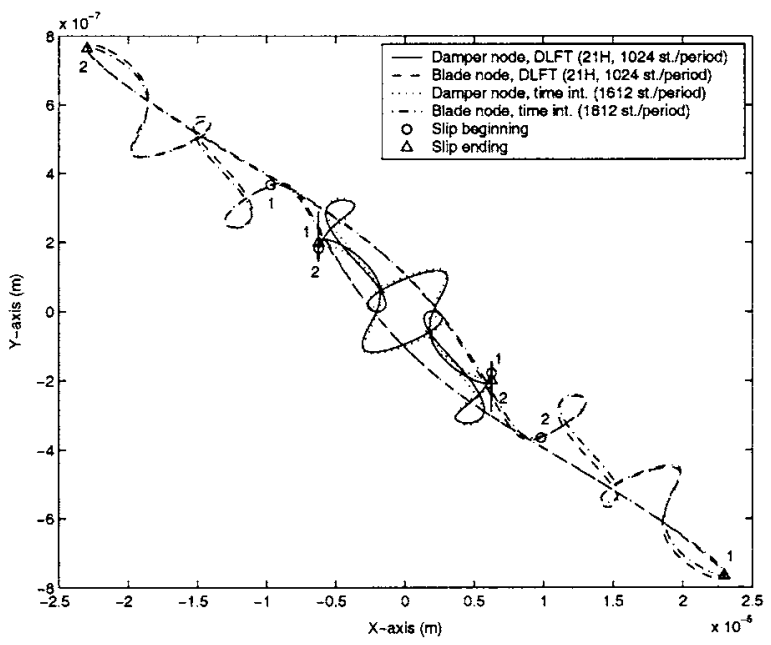

Fig. 10. Blade motion at the damper location, and damper motion, in the tangential plane, for case of a circular excitation $\left(\xi=0.01, \mu=0.1, \omega=124 \mathrm{~Hz}, F_{X}=50 \sin (\omega t), F_{Y}=50 \cos (\omega t), N_{h}=21\right.$ and $\left.\varepsilon=8 \times 10^{7}\right)$.

direction for the rectilinear excitation) and phase. The frequency responses (Figs. 9 and 13) show that these errors introduce a small frequency shift. It is hard to conclude that the DLFT is more accurate than the time integration of ABAQUS, although a problem in ABAQUS could be the precision in handling transitions. Indeed, an internally scaled constant is probably used in ABAQUS to detect numerically when the velocity is very close of zero. If this constant is too small, problems of convergence can occur. Namely, the constant drives the accuracy of the stickslip transitions and yields a small error. In the DLFT, no such constant is used to detect the transitions, and the accuracy is directly linked to the size of time step. 


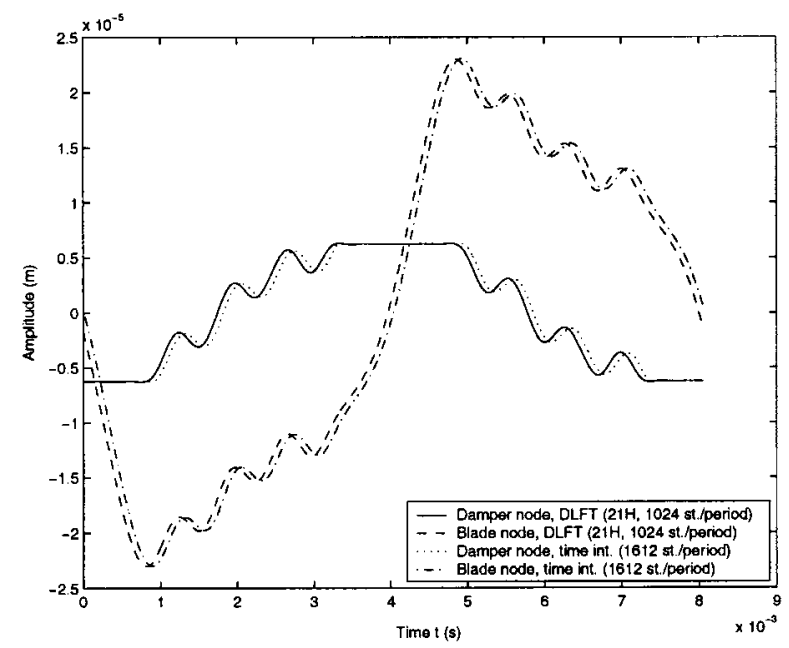

Fig. 11. Beam displacement at the damper location, and damper displacement, compared in the $X$ direction using the new DLFT method and time integration, for case of a circular excitation $\left(\xi=0.01, \mu=0.1, \omega=124 \mathrm{~Hz}, F_{X}=\right.$ $50 \sin (\omega t), F_{Y}=50 \cos (\omega t), N_{h}=21$ and $\left.\varepsilon=8 \times 10^{7}\right)$.

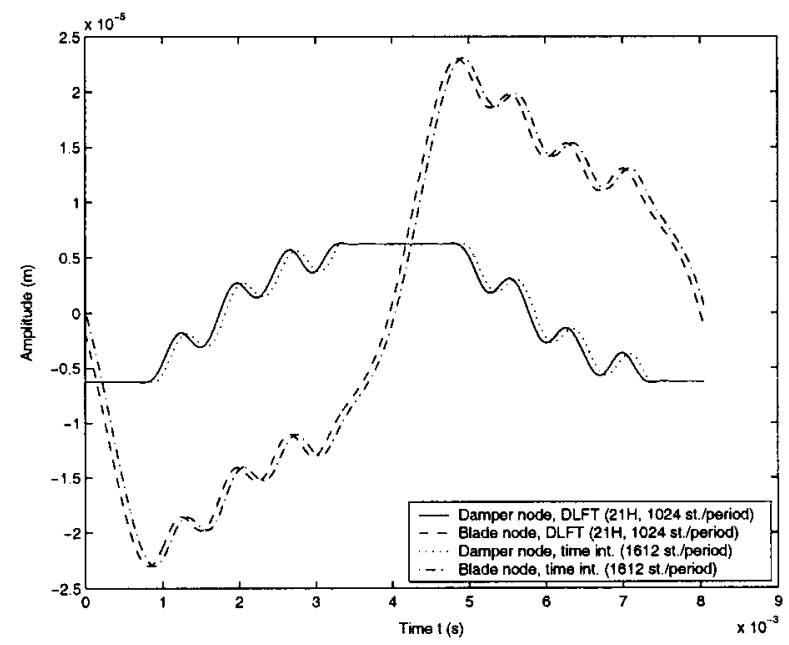

Fig. 12. Beam displacement at the damper location, and damper displacement, compared in the $Y$ direction using the new DLFT method and time integration, for case of a circular excitation $\left(\xi=0.01, \mu=0.1, \omega=124 \mathrm{~Hz}, F_{X}=\right.$ $50 \sin (\omega t), F_{Y}=50 \cos (\omega t), N_{h}=21$ and $\left.\varepsilon=8 \times 10^{7}\right)$.

In the third example, the response a mistuned system of 36 coupled beams (Fig. 14a) to a travelling wave excitation is considered.

The engine order of the excitation is three, corresponding to a phase change of $\pi / 6$ for the harmonic force on adjacent beams. The beam system has $7 \%$ random mistuning, which means 


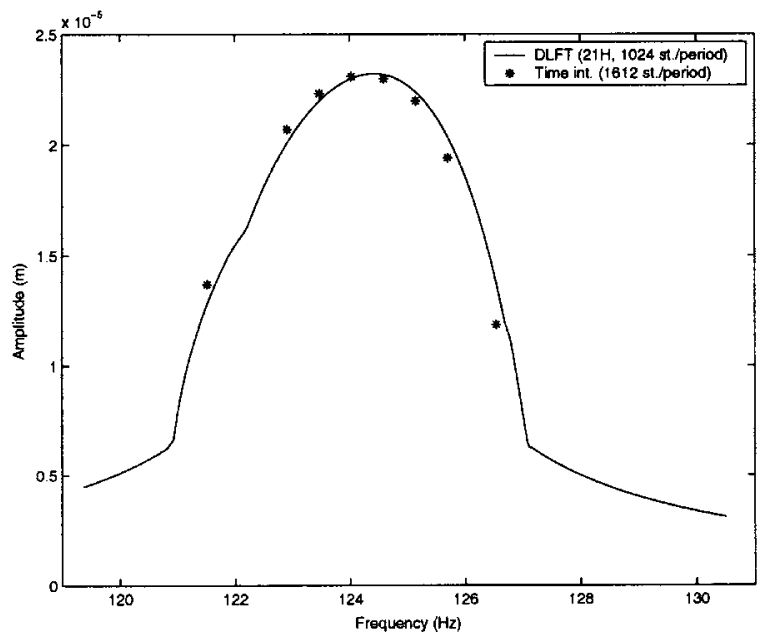

Fig. 13. Amplitude of the beam at the damper location for case of a circular excitation $(\xi=0.01, \mu=0.1, \omega=124 \mathrm{~Hz}$, $F_{X}=50 \sin (\omega t), F_{Y}=50 \cos (\omega t), N_{h}=21$ and $\left.\varepsilon=8 \times 10^{7}\right)$.
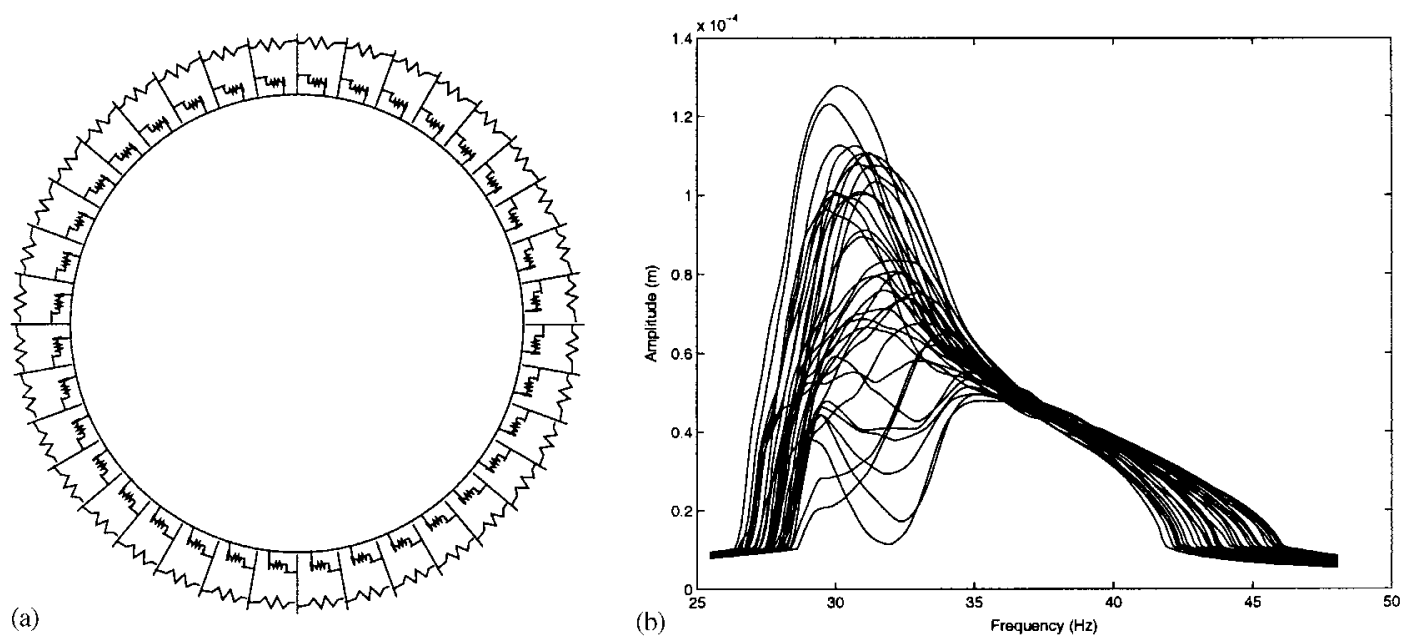

Fig. 14. (a) Bladed disk model with 36 beams, 36 ground-connected dampers, and 108 d.o.f.'s. (b) Beam displacements at the damper locations for a $7 \%$ mistuned 36-beam system. The coupling stiffness is $k_{c}=4500 \mathrm{~N} / \mathrm{m}$ and others parameters are given by $\xi=0.01, \mu=1, N_{h}=21$ and $\varepsilon=8 \times 10^{6}$.

that the individual beam natural frequencies are generated from a uniform random distribution with $7 \%$ standard deviation, in order to mimic differences caused by manufacturing tolerances. Each beam is condensed out using the Craig-Bampton component mode synthesis, retaining two active d.o.f.'s and the lowest first mode of free vibration of the beam fixed at the active d.o.f.'s. The mass and stiffness matrix of the beam components were provided by SNECMA Co. and are given in [32]. A coupling stiffness of $4500 \mathrm{~N}$ is applied between the free tips of two adjacent beams. There is no coupling through the disk in this particular system. One d.o.f. is retained for 
each friction damper, and the damper stiffness is $k=2.4 \times 10^{7} \mathrm{~N} / \mathrm{m}$. The normal pre-load applied to each damper is $246.048 \mathrm{~N}$ and the amplitude of the excitation on the free tip of each beam is $20 \mathrm{~N}$. Finally, the coefficient of friction is $\mu=1$.

The frequency response of the beam system is presented in Fig. 14b, where a viscous damping of $\xi=0.01$ is used for the beams and 21 harmonics are used in the DLFT.

Notice that no convergence problems were encountered, although a high level of accuracy was imposed. The purpose of this paper is not to carry out an extensive parametric study of mistuned systems, but rather to establish the validity of the DLFT on an arbitrarily mistuned large-scale system. However, one can at least note that in the chosen configuration, there are large differences in the peak resonant amplitudes of the various beams. For some frequencies, the factor between the beams response amplitudes can be as large as 10, although the system is only $7 \%$ mistuned. For this system, the blade magnification factor at the damper location, which is defined as the ratio of the maximum blade resonant amplitude for the mistuned system to the resonant amplitude for the tuned system, is 1.44 . This means that energy can be strongly localized to a few beams for such systems.

\section{Conclusions}

A new frequency-time domain method has been proposed for the efficient prediction of the steady state forced response of dry-friction-damped structural systems: the dynamic Lagrangian mixed frequency-time (DLFT) method. This formulation relies on the dynamic Lagrangians, defined as the non-linear contact forces obtained from the equations of motion in the frequency domain, with the adjunction of a penalization on the relative displacement at frictional interfaces calculated two different ways: first, the interface displacements are obtained in the frequency domain from the non-linear solver; second, they are calculated in the time domain using Coulomb friction and non-penetration criteria for determining the states at the interfaces. Note that a penalty procedure on the relative velocities could be carried out in the same way, although its application in the frequency domain would be limited to systems with a known mean position. Furthermore, with simple modifications, the DLFT formulation could be used in dynamic problems of contact between elastic and rigid structures.

The DLFT method allows one to suppress the springs generally used in the definition of contact elements in time and frequency domain methods. This means that finite element models can be used readily, without having to develop a special model for the frictional interfaces. Furthermore, the general case of three-dimensional motion at frictional interfaces can be easily handled with the DLFT method. This new approach retains the advantages of a time-integration procedure, which uses Lagrange multipliers for example, while providing fast and accurate calculation of the steady state forced response. The robustness of the algorithm and the lack of convergence problems should also be noted, which makes the method suitable for large-scale systems.

Since it does not require special modelling of the contact interfaces, the DLFT method has the potential to be readily applicable to complex friction interface configurations such as shrouds and dovetail attachments in bladed disks. 


\section{Acknowledgements}

This study was carried out for the SNECMA Co. The authors gratefully acknowledge the Société Nationale d'Étude et de Construction de Moteurs d'Aviation (SNECMA) for its support.

\section{References}

[1] C. Pierre, A, Ferri, E. Dowell, Multi-harmonic analysis of dry friction damped systems using an incremental harmonic balance method, Journal of Applied Mechanics 51 (4) (1985) 958-964.

[2] S. Lau, Y. Cheung, S. Wu, Incremental harmonic balance method with multiple time scales for aperiodic vibration of nonlinear systems, Journal of Applied Mechanics 50 (1983) 871-876.

[3] J.H. Wang, W.K., Chen, Investigation of the vibration of a blade with friction damper by HBM, Transactions of the American Society of Mechanical Engineers, Journal of Engineering for Gas Turbines and Power 115 (1993) 294-299.

[4] T. Cameron, J. Griffin, An alternating frequency/time domain method for calculating the steady-state response of nonlinear dynamic systems, Journal of Applied Mechanics 56 (1989) 149-154.

[5] J. Guillen, C. Pierre, An efficient, hybrid, frequency-time domain method for the dynamics of large-scale dry-friction damped structural systems, in: F. Pfeiffer, Ch. Glocker (Eds.), IUTAM Symposium on Unilateral Multibody Contact, Kluwer Academic Publishers, Dordrecht, 1999.

[6] O. Poudou, Modeling of friction damping in bladed disk assemblies, Rapport de fin d'études, Ecole Centrale de Nantes, France, 1999.

[7] B.S. Garbow, K.E. Hillstrom, J.J. More, User Guide for Minpack-1, National Argonne Laboratory, Illinois, 1980.

[8] M.J.D. Powell, A hybrid method for nonlinear equations, in: P. Rabinowitz (Ed.), Numerical Methods for Nonlinear Equations, Gordon and Breach, London, UK, 1969.

[9] J.H. Griffin, Friction damping of resonant stresses in gas turbine engine airfoils, Journal of Engineering for Power 102 (1980) 329-333.

[10] W.E. Whiteman, A.A. Ferri, Analysis of beam-like structures with displacement-dependent friction forces: Part I: single-degree-of-freedom model, Proc. of ASME 1995 Design Engineering Technical Conferences, Symp. on Friction Damping and Friction-Induced Vibration, Vol. 3(A) 1995, pp. 1093-1100.

[11] W.E. Whiteman, A.A. Ferri, Analysis of beam-like structures with displacement-dependent friction forces: Part II: multi-degree-of-freedom model, Proc. of ASME 1995 Design Engineering Technical Conferences, Symp. on Friction Damping and Friction-Induced Vibration, Vol. 3(A) 1995, pp. 1101-1108.

[12] K.Y. Sanliturk, M. Imregun, D.J. Ewins, Harmonic balance vibration analysis of turbine blades with friction dampers, Journal of Engineering for Power 119 (1997) 96-103.

[13] A.A. Ferri, B.S. Heck, Vibration analysis of dry friction damped turbine blades using singular perturbation theory, Journal of Engineering for Gas Turbines and Power, Transactions of the American Society of Mechanical Engineers 120 (1998) 588-595.

[14] C.H. Menq, J.H. Griffin, J. Bielak, The influence of a variable normal load on the forced vibration of a frictionally damped structure, Journal of Engineering for Gas Turbines and Power, Transactions of the American Society of Mechanical Engineers 108 (1986) 300-305.

[15] B.D. Yang, M.L. Chu, C.H. Menq, Stick-slip-separation analysis and non-linear stiffness and damping characterization of friction contacts having variable normal load, Journal of Sound and Vibration 210 (4) (1998) $410-481$.

[16] C.H. Menq, B.D. Yang, Non-linear spring resistance and frictional constraint having two-dimensional motion, Journal of Applied Mechanics 56 (1989) 149-154.

[17] C.H. Menq, P. Chidamparam, J.H. Griffin, Friction damping of two-dimensional motion and its application in vibration control, Journal of Sound and Vibration 144 (3) (1991) 427-447.

[18] K.Y. Sanliturk, D.J. Ewins, Modelling two-dimensional friction contact and its application using harmonic balance method, Journal of Sound and Vibration 193 (2) (1996) 511-523. 
[19] B.D. Yang, J.H. Griffin, Characterization of 3D contact kinematics and prediction of resonant response of structures having 3D frictional constraint, Journal of Sound and Vibration 217 (5) (1998) 909-925.

[20] B.D. Yang, C.H. Menq, Characterization of contact kinematics and application to the design of wedge dampers in turbomachinery blading: Part 1, stick-slip contact kinematics, Journal of Engineering for Gas Turbines and Power 120 (1998) 410-417.

[21] B.D. Yang, C.H. Menq, Characterization of contact kinematics and application to the design of wedge dampers in turbomachinery blading: Part 2, prediction of forced response and experimental verification, Journal of Engineering for Gas Turbines and Power 120 (1998) 418-423.

[22] C.H. Menq, J. Bielak, J.H. Griffin, The influence of microslip on vibratory response, Part I: a new microslip model, Journal of Sound and Vibration 107 (2) (1986) 279-293.

[23] C.H. Menq, J.H. Griffin, J. Bielak, The influence of microslip on vibratory response, Part II: a comparison with experimental results, Journal of Sound and Vibration 107 (2) (1986) 295-307.

[24] G. Csaba, Friction damping of turbine blade vibrations using a microslip model, Journal of Machine Vibration 4 (1995) 2-7.

[25] E.J. Berger, M.R. Begley, M. Mahajani, Structural dynamic effects on interface response: formulation and simulation under partial slipping conditions, Journal of Applied Mechanics 67 (2000) 785-792.

[26] T.A. Laursen, J.C. Simo, A continuum-based finite element formulation for the implicit solution of multibody, large deformation frictional contact problems, International Journal for Numerical Methods in Engineering 36 (1993) 3451-3485.

[27] J.-H. Heegaard, A. Curnier, An augmented Lagrangian method for discrete large-slip contact problems, International Journal for Numerical Methods in Engineering 36 (36) (1993) 569-593.

[28] P. Wriggers, G. Zavarise, Application of augmented Lagrangian techniques for non-linear constitutive laws in contact interfaces, Communications in Numerical Methods in Engineering 9 (1993) 815-824.

[29] T.A. Laursen, B.N. Maker, An augmented Lagrangian quasi-Newton solver for constrained nonlinear finite element applications, International Journal for Numerical Methods in Engineering 38 (1995) 3571-3590.

[30] ABAQUS Standard, User's Manual, Vol. II, Version 5.6, Hibbitt, Karlsson \& Soresen, Inc., Pawtucket, RI, USA, 1996.

[31] R. Craig, M. Bampton, Coupling of substructures for dynamic analysis, American Institute of Aeronautics and Astronautics Journal 8 (7) (1968) 1313-1319.

[32] M. Berthillier, C. Dupont, R. Mondal, J.J. Barrau, Bladed forced response analysis with friction dampers, Journal of Engineering for Gas Turbines and Power 120 (1998) 468-474. 